\title{
Review of: "The impact of COVID-19 on private and public primary care physicians: A cross-sectional study"
}

Neil Holtzman ${ }^{1}$

1 Johns Hopkins University

Potential competing interests: The author(s) declared that no potential competing interests exist.

I am grateful to the organizers of Qeios for inviting my comments on the paper by Jared Lau et al on the impact of COVID-19 on primary care physicians (PCPs).[1] They surveyed two groups of PCPs in Singapore during the Covid-19 pandemic, the majority in private practice, the minority in government-subsidized group practice polyclinics. Their findings, as the authors say, "cover common themes that may have significant implications for other healthcare systems around the world." It is these implications that I will consider, particularly the authors' contention that "it is critical that [private] PCPs continue to be supported in their front line role." A periodic, ongoing survey of primary care providers in the U. S. covers similar ground.[2] Lau et al acknowledge that their low response rates (4\% for private PCPs; $29 \%$ for public PCPs) could have resulted in selection bias.

Most PCPs in both groups agreed that that they were at high risk of Covid-19 exposure (89.3\% private vs. $87.0 \%$ public PCPs), but that such risks were part of their profession. Private PCPs were more likely to agree that they should not be caring for COVID-19 patients (19.4\% private vs. $7.2 \%$ public) and that their COVID-19 exposure risk was unacceptable ( $13.6 \%$ private vs. $4.3 \%$ public). Adjusted for gender, ethnicity, and years of medical experience, both differences were statistically significant ( $<<0.05$ ), as were differences in factors that could explain private PCPs' greater reluctance to care for COVID-19 patients: being less likely to receive training for infection control at the workplace; less likely to have a source of advice for the use of personal protective equipment; and less confident that their workplaces were prepared or had a preparedness plan.[*] That "private PCPs are often self-employed and their absence from work as a result of contracting CovID-19 could be detrimental to their livelihoods" could also explain their greater reluctance to care for COVID-19 patients or accept the risks of exposure. Regarding self-employed private PCPs:, Lau et al. comment, "(T)heir absence from work could be detrimental to their livelihoods. This has been reflected locally and in other healthcare systems, where private GPs have also suffered a tremendous loss in revenue as patients deferred primary care visits to reduce risk of COVID-19 transmission." Sixty percent of their respondents were private PCPs "who operate their own general practices (GP) or family medicine clinics (FMC)," suggesting they are self-employed,

Lau at al. conclude that "the important role of governmental support for both private and public PCPs in pandemics is apparent from our findings." The early success of Singapore in curbing the COVID-19 pandemic, however, had more to do with "a hard-line "circuit-breaker' lockdown"[3] than with practicing PCPs.

In the United States where I live, "Almost seven in 10 [70\%] of physicians are now employed by hospitals or corporations like private equity firms and health insurers." PCPs in this category are "employee PCPs," not "private PCPs." Members of the Physicians Advocacy Group, which commissioned the U.S. study, "are concerned about the loss of physician autonomy...associated with companies that employ physicians..."[4]

The increase of non-autonomous employee PCPs in the U.S., many of whom work for large healthcare networks, has advantages and disadvantages. Compared to private PCPs these networks can afford to purchase personal protective equipment for their employees and expand the use of telemedicine, which happened during the pandemic.. On the other hand, providing optimal primary care may not be in the best financial interests of commodified healthcare networks..

Like many others, the healthcare network to which I belong as a patient has partially abdicated the gatekeeper-first contact care function of primary care, as have other networks. When I sign-in online, the first message on my screen is, "Call $9 * 1 * 1$ if you have an emergency." If I phone my PCP, the recorded response is, "If you have a lifethreatening emergency, hang up and dial $9 * 1 * 1$." If I as a retired physician cannot always tell whether my symptoms are life-threatening, how can the multitudes without medical training be expected to know?[†]

Emergency departments in the U.S. saw a $60 \%$ increase in the percentage of deaths among $9 * 1 * 1$-initiated patient contacts by Emergency Medical Services between the weeks of March 14, 2020 and April 11 (1.5\% to 2.4\%),[5] coinciding with the beginning of the pandemic, and with the abrupt shutdown of non-emergency ambulatory care centers. The chaos wrought by the shutdown, followed by makeshift and often inefficient and undependable CovID-19 testing services,[6] and the fear engendered, is not something I would like to experience again.

The organization of primary care in the U.S., which ranks first of 224 nations in total COVID-19 cases (146,000/1M pop), and elsewhere, including Singapore (which ranks 85 th (43,100 cases/1M pop),[7] is not prepared for the arrival of the next health disaster whether it is due to a hitherto unknown viral pathogen, a nuclear accident, or massive chemical contamination of air, water, or food. Let's look at what's needed in four sequential stages, paying particular attention to the role of PCPs.

Recognition. At this first stage, PCPs will play an important role in recognizing increased numbers of patients with unusual symptoms or an unanticipated increase of patients with common symptoms. Reporting them to local health departments that are part of an organizational public health pyramid capable of assessing new cases epidemiologically is essential.[f] At the apex of the pyramid stand organizations like the Centers for Disease Control and Prevention (CDC) nationally and the World Health Organization (WHO) globally. They have the responsibility of establishing the nature and magnitude of the underlying condition and declaring a public health emergency 
when necessary. PCPs should also be collecting biological specimens and sending them to appropriate laboratories for evaluation and aiding appropriate specialists in assessing the natural history of the condition. These activities should operate in cooperation with the public health pyramid and the findings should be shared through prompt publication leading to further validation by replication and other means.

Mobilization. As information on the nature and magnitude of the threat is being gathered, organizations have to prepare for the worst-case scenario. Stockpiling personal protective equipment, improving other barriers to infection, radiation, mass poisonings, or other potential harms, and having adequate supplies of equipment needed to manage an increased number of life-threatening situations, are beyond the scope of individual PCPs, requiring investments that only large organizations can afford, especially when they may never be used; government assistance is likely to be needed. Because people will continue to get or be sick from other causes, efforts should be made to maintain essential aspects of non-crisis care, including primary care. These may involve innovative ways of evaluating victims, such as telemedicine, and separating contagious patients from patients with other immediate healthcare needs.

Once the viral or other toxic agent has been identified, developing the ability to detect affected individuals by diagnostic tests becomes important, particularly if there is a pre-symptomatic stage of the condition that can be prevented from advancing or spreading to others. The development and production of vast amounts of new drugs to treat, and vaccines to prevent, the condition will almost certainly need government funding. Regulation by government agencies like the Food and Drug Administration will be required to assure their safety and effectiveness.

Intervention. In crises on the scale of COVID-19, PCPs can and should be involved in diagnostic, therapeutic, and preventive activities but they cannot do so without help as they continue their other essential activities. They can educate about the importance of lockdowns, safe distancing, and wearing masks but they cannot enforce rules requiring them. When person-to-person spread is at work, contact tracing to identify, quarantine, and possibly treat early cases requires more workers than PCPs can provide; expanding the public health work force could meet this need and also that of monitoring the progression of the crisis, such as new cases, hospitalizations, deaths, and vaccination rates. Recruiting pharmacists to administer vaccines, and mounting special campaigns may also be needed, as might government mandates to eliminate the condition.

Stabilization. Eventually the crisis will be alleviated and activities essential to the wellbeing of the community, such as education and employment can be fully resumed Material resources needed at the height of the pandemic such as personal protective equipment, as well as human resources, such as contact tracers, will no longer be needed. As time passes the lessons may be forgotten. Governments have the institutional memory and the resources to make sure mistakes in the past are not repeated.

$$
\text { * }
$$

As important as primary care is in day-to-day healthcare as well as in recognizing impending health crises for which people seek their care, and in later stages when they can diagnose, treat, and immunize, crises will not be overcome without government intervention to provide adequate supplies, including drugs and vaccines, excess manpower, temporary facilities for isolation of cases, and without laws and regulation to ensure an optimal public response. Governments alone have the wherewithal to provide assistance when mass unemployment ensues and small businesses are forced to shut down. It is in that context that support for self-employed PCPs might be needed, as in Singapore, not because they play an "important front line role."

The increased commodification of healthcare in the United States has reduced the importance of self-employed PCPs. The chaotic shutdown of ambulatory facilities in both for-profit and non-profit healthcare networks limited the ability of "employee PCPs" to play an adequate role in recognition and intervention.

That brings me, finally, to "public PCPs." In Singapore, a higher proportion of them than of private PCPs accepted the risks of caring for COVID-19 patients and felt better prepared, perhaps because they work in government-subsidized health clinics that provide consistent guidance to protect the public's health. Every country needs more of them.

Footnotes

[*] Unfortunately, the authors did not examine in bivariate analysis or multiple regression whether these factors affected private PCPS willingness to care for COVID-19 patients or their acceptance of the risk of their exposure to COVID-19.

[†] I would feel better about needing to go to the Emergency Room if I heard it from my PCP rather than from a recorded message. The only way I can reach my PCP when I perceive I might have an emergency is to lie and leave a message. Usually, I won't get a response for several hours or until the next day, by which time I might die if my condition were truly life-threatening.

[f]This doesn't always happen. PCPs in and around Old Lyme Connecticut in the early 1970s failed to report an increased frequency of juvenile arthritis often associated with an unusual rash. It was only when two mothers notified the Connecticut Department of Health and Yale School of Medicine of the unusual cluster that investigations leading to the discovery of Lyme disease were initiated. (see Elbaum-Garfinkle S. "Close to home: A history of Yale and Lyme disease." Yale J Biol Med. 2011 Jun; 84(2): 103-

108. https://www.ncbi.nlm.nih.gov/pmc/articles/PMC3117402/

References

[1] Lau J, DHY, Wong GJ et al. "The impact of COVID-19 on private and public primary care physicians: A cross-sectional study. J Infect Pub HIth 2021.

14:285-289. https://doi.org/10.1016/j.jiph.2020.12.028 
[2] Primary care collaborative. Primary care \& COVID-19. 2021. https://www.pcpcc.org > covid

[3]Rising D, Liang A. Singapore strategy of living with COVID raises concern, hope. AP News. 30 Sept. 2021. https://apnews.com/article/coronaviruspandemic-business-health-pandemics-singapore-9d1ae27b0b79294924c5765b5ba619d2

[4] Bannow T. Nearly 70\% of U.S. physicians now employed by hospitals or corporations, report finds. Modern Healthcare. 20 June 2021. https://www.modernhealthcare.com/providers/nearly-70-us-physicians-now-employed-hospitals-or-corporations-report-finds? adobe_mc =MCMID\%3D48073835290402149623555742978784066735\%7CMCORGID\%3D138FFF2554E6E7220A4C98C6\%2540AdobeOrg\%7CTS\%3D163754

[5] Lerner EB, Newgard CD, Mann NC. Effect of the Coronavirus Disease 2019 (COVID-19) Pandemic on the U.S. Emergency Medical Services System: A Preliminary Report. Acad Emer Med. 2020. 27(8):693-699. https://doi.org/10.1111/acem.14051

[6]Holtzman NA. Invited commentary: The covid-19 pandemic in. the United States. Intl J Equity HIth 2021 20:3. https://doi.org/10.1186/s12939-02001354-6

[7]WORLDOMETER. 18 Nov. 2021. https://www.worldometers.info/coronavirus/\#main_table 